\title{
Some properties of solutions of elliptic partial differential equations of the second order
}

\author{
by JAN BOCHENEK (Kraków)
}

Let $D$ be a domain in the space of $m$ variables $X\left(x_{1}, \ldots, x_{m}\right)$. Assume that the boundary $F(D)$ of $D$ or at least a closed part $\Sigma$ of $F(D)$ is of class $C^{2}$, i.e. $F(D)$ or $\Sigma$ is given by the equation $G\left(x_{1}, \ldots, x_{m}\right)=0$, where the function $G$ is of class $C^{2}$ in an $m$-dimensional domain containing $F(D)(\Sigma)$ and, moreover, $\operatorname{grad}^{2} G>0$. Let

$$
\varepsilon(u) \equiv \sum_{i, j=1}^{m} a_{i j}(X) \frac{\partial^{2} u}{\partial x_{i} \partial x_{j}}+\sum_{k=1}^{m} b_{k}(X) \frac{\partial u}{\partial x_{k}}+c(X) u=f(X),
$$

where the coefficients $a_{i j}(X)=a_{j i}(X), b_{k}(X) \quad(i, j, k=1, \ldots, m), c(X)$ and the function $f(X)$ are defined and bounded in the closure $\bar{D}$ of a bounded domain $D$. Let the characteristic form $\sum_{i, j=1}^{m} a_{i j}(X) \lambda_{i} \lambda_{j}$ of equation (1) be uniformly positive definite in $D$. We shall prove

LEMma 1. If in the domain $\bar{D} 1^{\circ} f(X) \geqslant 0$ (or $\left.f(X) \leqslant 0\right), 2^{\circ}$ a part of $F(D)$ contained in a neighbourhood of a point $X_{0}$ of $\Sigma$ belongs to $\Sigma$, $3^{\circ} u(X)$ is a regular solution of (1) (for the definition of the regular solution of (1), see [3], p. 118) in the closure $\bar{D}$ of $D$ such that $u\left(X_{0}\right)=0$ and $u(X)<0$ (or $u(X)>0$ ), then

$$
\limsup _{X \rightarrow X_{0}} \frac{u(X)-u\left(X_{0}\right)}{\left|X-X_{0}\right|}<0 \quad\left(\text { or } \quad \liminf _{X \rightarrow X_{0}} \frac{u(X)-u\left(X_{0}\right)}{\left|X-X_{0}\right|}>0\right),
$$

where $X$ belongs to an open rectilinear segment contained in $D$ such that the segment ends at the point $X_{0}$ and is not tangent to $F(D)$ at $X_{0}\left(X \rightarrow X_{0}\right.$ along a half-straightline "penetrating" into the interior of $D$ ).

Proof. Take the transformation

$$
u(X)=v(X) W(X)
$$

where

$$
W(X)=\prod_{i=1}^{m} \cos \mu\left(x_{i}-\grave{x}_{i}\right), \quad X_{0}\left(\stackrel{\circ}{x}_{1}, \ldots, \stackrel{\circ}{x}_{m}\right)
$$


Then (1) takes the form (for $W(X) \neq 0$ )

$$
\bar{\varepsilon}(v) \equiv \sum_{i, j=1}^{m} a_{i \jmath}(X) \frac{\partial^{2} v}{\partial x_{i} \partial x_{j}}+\sum_{k=1}^{m} \bar{b}_{k}(X) \frac{\partial v}{\partial x_{k}}+\bar{c}(X) v=\bar{f}(X) .
$$

where $\bar{c}(X)=\frac{1}{W(X)} \varepsilon(W), \bar{f}(X)=\frac{1}{W(X)} f(X)$.

We shall show that there exist a number $\mu$ and a neighbourhood $O\left(X_{0}\right)$ of $X_{0}$ such that $\bar{c}(X)<0$ and $W(X)>0$ in $O\left(X_{0}\right) \cap \bar{D}$. Indeed, obvious computations show that

$$
\frac{1}{W(X)} \mathcal{E}(W)=-\mu^{2} g(X, \mu)-\mu h(X, \mu)+o(X),
$$

where

$$
\begin{aligned}
& g(X, \mu)=\sum_{i=1}^{m} a_{i i}(X)-\sum_{\substack{i, j=1 \\
(i \neq j)}}^{m} a_{i j}(X) \operatorname{tg} \mu\left(x_{i}-\ddot{x}_{i}\right) \operatorname{tg} \mu\left(x_{j}-\stackrel{\circ}{x}_{j}\right) \\
& h(X, \mu)=\sum_{k=1}^{m} b_{k}(X) \operatorname{tg} \mu\left(x_{k}-\stackrel{\circ}{x}_{k}\right) .
\end{aligned}
$$

By our assumptions $\sup _{\boldsymbol{X} \in D}|c(X)|=M<\infty$. Observe that if the inequality

$$
-\mu^{2} g(X, \mu)-\mu h(X, \mu)+M<0
$$

holds, then also $\varepsilon(W)<0$ holds. For $\mu \neq 0$ (5) is equivalent to

$$
-g(X, \mu)-\frac{h(X, \mu)}{\mu}+\frac{M}{\mu^{2}}<0 .
$$

Take $\mu$ such that

$$
\frac{4 M}{\mu^{2}}<\inf _{X \in D}\left[\sum_{i=1}^{m} a_{i i}(X)\right]=A \quad(A>0),
$$

and a neighbourhood $O\left(X_{0}\right)$ such that

$$
\frac{h(X, \mu)}{\mu}>-\frac{A}{4} \quad \text { and } \quad g(X, \mu)>\frac{3}{4} A, \quad W(X)>0 .
$$

Then (6) is satisfied, which implies that $\bar{c}(X)<0$ and $\bar{f}(X) \geqslant 0$ (or $\bar{f}(X) \leqslant 0)$ for $X \in O\left(X_{0}\right) \frown \bar{D}$.

Since the boundary $F(D)$ of $D$ is of class $C^{2}$ at $X_{0}$, then, as we know (see [1]), there exists a ball $K$ whose boundary $F^{\prime}(K)$ is tangent to $F(D)$ at $X_{0}$ and whose other points all belong to $D$. In the common part $\Omega$ 
of $O\left(X_{0}\right)$ and $K$ the function $v$ satisfies all the assumptions of a lemma of O. A. Olejnik ([5]). Therefore

$$
\left.\limsup _{X \rightarrow X_{0}} \frac{v(X)-v\left(X_{0}\right)}{\left|X-X_{0}\right|}<0 \quad \text { (or } \quad \liminf _{X \rightarrow X_{0}} \frac{v(X)-v\left(X_{0}\right)}{\left|X-X_{0}\right|}>0\right),
$$

where $X \rightarrow X_{0}$ along a rectilinear segment contained in $\Omega$ and non-tangent to $F(\Omega)$.

On the other hand, since $u\left(X_{0}\right)=v\left(X_{0}\right)=0$, so

$$
\frac{u(X)-u\left(X_{0}\right)}{\left|X-X_{0}\right|}=\frac{v(X)-v\left(X_{0}\right)}{\left|X-X_{0}\right|} W(X) .
$$

Observe that

$$
\lim _{x \rightarrow X_{0}} W(X)=1 \text {. }
$$

Lemma 1 now follows from (7), (8) and (9).

THEOREM 1. If in equation (1) $f(X) \geqslant 0$ (or $f(X) \leqslant 0$ ) for $X \in D$, and if $u(X)$ is an arbitrary non-positive (or non-neativeg) solution of (1) regular in the closure $\bar{D}$ of $D$ such that $\sup u(\bar{D})=0 \quad($ or $\inf u(\bar{D})=0)$, then $u(X)$ vanishes at a point of $F(D)$. Moreover, if $u(X)$ also vanishes at a point in the interior of $D$, then $u(X)$ vanishes identically in $D$.

Proof. By Lemma 1 the proof of Theorem 1 is analogous to the proof of E. Hopf's theorem (see [2], [4]).

Theorem 2. If $1^{0}$ the boundary $F^{\prime}(D)$ of $D$ is of class $C_{\sigma}^{1}\left({ }^{1}\right), 2^{0}$ there is no tangent plane to $F(D)$ at $X_{0} \in F(D), 3^{\circ}$ there is a ball $K$ belonging to $D$ such that boundary $F^{\prime}(K)$ of $K$ contains the point $X_{0}, 4^{0} u(X)$ is a biregular $\left(^{2}\right)$ solution of (1) with $f(X) \equiv 0$ such that $u(X) \neq 0$ in $D$ and $u(X)=0$ on $F(D)$, then $u(X)$ changes its sign in every neighbourhood of $X_{0}$.

Proof. Suppose there is a neighbourhood $O\left(X_{0}\right)$ of $X_{0}$ contained in $D$ such that $u(X) \neq 0$ in $O\left(X_{0}\right) \cap D$. Then, by Lemma 1 , the derivative of $u(X)$ in an arbitrary direction non-tangent to the boundary of $K$ at $X_{0}$ is different from zero.

On the other hand, since there is no tangent plane to $F(D)$ at $X_{0}$, then there are $m$ linearly independent vectors tangent to $F(D)$ at $X_{0}$. It is obvious that the derivative of $u(X)$ in each direction tangent to $F(D)$ at $X_{0}$ is equal to zero. This, however, implies (comp. [3], p. 44) that $\partial u / \partial x_{i}=0(i=1, \ldots, m)$ at $X_{0}$. Thus all the derivatives of $u(X)$ in directions non-tangent to $F(D)$ at $X_{0}$ are equal to zero. We have got a contradiction. Thus the proof is complete.

(1) For a definition of a surface of class $C_{\sigma}^{2}$, see [3], p. 132.

$\left({ }^{2}\right)$ For a definition of a biregular solution of (1), see [3], p. 118. 
The following corollaries are simple consequences of Theorems 1 and 2 .

Corollary 1. If the boundary $F(D)$ of $I D$ satisfies $1^{\circ}, 2^{\circ}$ aud $3^{\circ}$ of Theorem 2, then a function $u(X)$ satisfying (1) with $f(X) \equiv 0$ such that $u(X)=0$ on $F(D)$ and $u(X)$ does not change its sign in $D$ cannot be of class $C^{1}$ in the closure $\bar{D}$ of $D$.

Corollary 2. If $1^{0} u\left(X_{0}\right)=0$ and $d u / d l=0$ at $X_{0} \in F^{1}(D), l$ denoting a non-tangent direction to $F(D)$ at $X_{0}$ "penetrating" into the interior of $D$, $2^{\circ}$ there exists a ball $K$ contained in $D$ such that its boundary $F^{\prime}(K)$ contains $X_{0}, 3^{\circ}$ the function $u(X)$ is a non-zero solution of equation (1) with $f(X) \equiv 0,4^{0}$ the $u(X)$ is of class $C^{1}$ in the closure $\bar{D}$ of $D$, then the function $u(X)$ changes its sign in every component of the common part of $D$ and an arbitrary neighbourhood of $X_{0}$.

Corollary 3. If $Z$ denotes the set of zeros of $u(X)$, where $u(X)$ is a non-zero solution of (1) with $f(X) \equiv 0$, then $1^{\circ} Z$ contains no isolated points, $2^{\circ}$ the function $u(X)$ changes its sign in every neighbourhood of an arbitrary point of $Z, 3^{\circ}$ the set $Z$ divides the domain $D$, i.e. in every neighbourhood of an arbitrary point of $Z$ there exist points belonging to at least two different components of $D-Z$.

CoRollary 4. If at a point $X_{0}$ belonging to the interior of $D$ we have $u\left(X_{0}\right)=0$ and $\operatorname{grad}^{2} u\left(X_{0}\right)=0$, then the point $X_{0}$ is a "ramification" point of the surface $u(X)=0$ in the sense that every ball contained in $D$ such that its surface touches $X_{0}$ contains points of the surface $u(X)=0$.

This paper has been prepared under the guidance of professor M. Krzyżański. I am very much obliged to him for that.

\section{References}

[1] S. Goląb i M. Kucharzewski, O polożeniu kul stycznych do powierzchni, Prace Mat. 3 (1959), pp. 167-184.

[2] E. Hopf, Elementare Betrachtung über die Losungen partieller Differentialgleichungen zweiter Ordnung von elliplischen Typus, Sitzungsberichte Preuss. Akad. Wiss. 19 (1927).

[3] M. Krzyżański, Równania różniczkowe czastkowe rzędu drugiego, vol. I, Warszawa 1957.

[4] C. Miranda, Sulla proprieta di minimo e di massimo..., Atti. Acad. Naz. Lincei 8 (Ser. 10) (1951).

[5] О. А. Олейник (O. A. Olejnik), О эадаче Дирихле для уравнений эллиптического muna, Матем. сб. 24 (66) (1949), pp. 3.14. 